\title{
Preparation of cooled and bunched ion beams at ISOLDE-CERN
}

\author{
I. Podadera ${ }^{1,2, a}$, T. Fritioff ${ }^{1}$, A. Jokinen ${ }^{1,3,5}$, J.F. Kepinski ${ }^{4}$, M. Lindroos ${ }^{1}$, D. Lunney ${ }^{4}$, and F. Wenander ${ }^{1}$ \\ 1 CERN, AB department, CH-1211 Geneva 23, Switzerland \\ 2 Universitat Politècnica de Catalunya, Barcelona, Spain \\ 3 Department of Physics, FIN-40014 University of Jyväskylä, Jyväskylä, Finland \\ 4 CSNSM-IN2P3-CNRS, F-91405 Orsay-Campus, France \\ ${ }^{5}$ Helsinki Institute of Physics, FIN-00014 University of Helsinki, Helsinki, Finland
}

Received: 12 December 2004 / Revised version: 26 January 2005 /

Published online: 10 May 2005 - (c) Società Italiana di Fisica / Springer-Verlag 2005

\begin{abstract}
At ISOLDE a new RadioFrequency Quadrupole ion Cooler and Buncher (RFQCB) is being constructed to improve ion optical properties of low-energy RIBs. The new features of the mechanical design and the status of the test bench, which will serve to test the device, will be presented in this contribution.
\end{abstract}

PACS. 41.85.-p Beam optics

\section{Introduction}

The new Radio Frequency Quadrupole ion Cooler and Buncher (RFQCB) for ISOLDE [1], called ISCOOL (ISolde COOLer) will be able to cool most ion beams delivered by the High-Resolution Separator (HRS) (see $[2,3]$ ). Therefore, ISCOOL will be completely integrated at ISOLDE and will be able to deliver most of different ion beams coming out from HRS to the experiments working with this separator. Unlike others existing similar devices $[4,5]$ with more specific working conditions, ISCOOL is defined as a general purpose ion trap for the preparation of cooled and bunched radioactive ion beams. This sets special requirements for flexibility and reliability of the device.

One of the main points to assure the flexibility of the system is to design a robust and simple mechanical structure. Some of the highlights of the design are presented in the following section. In addition, the reliability and performance of the system have to be tested. A new test bench has been constructed with this objective and the first results will be reported in the last section.

\section{Mechanical construction of the cooler}

The mechanical design of the RFQCB provides some unique features in comparison with the devices constructed before. The main points to be underlined are: the axial electrodes (see fig. 1), which allow a simplified electronics system separating the RF and DC components; a

\footnotetext{
${ }^{a}$ Conference presenter; e-mail: Ivan.Podadera@cern.ch
}
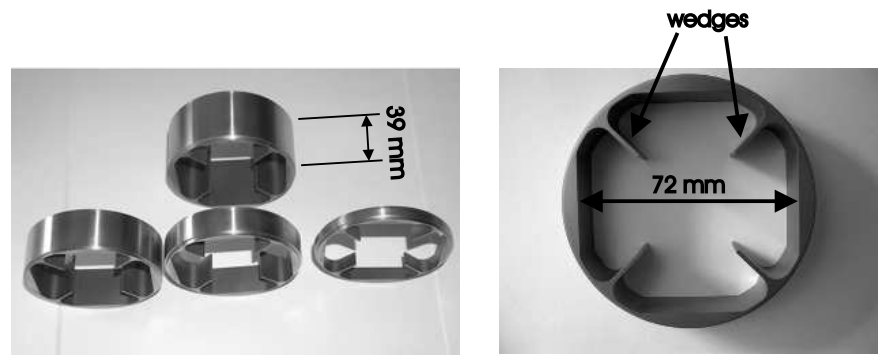

Fig. 1. Picture of axial electrodes of different lengths (left) and axial view showing the four-wedges shape (right).

mechanical structure of variable-depth, stacked axial electrodes forming a cavity which encloses the buffer gas volume and accommodates (separate) RF-rods, providing a flexible system for the optimization of the axial electric field of the cooler (see [6] for further information). Once all the parts are received from the workshops, mechanical compatibility will be verified in a full assembly of the apparatus.

\section{Test bench}

Before installation on-line at ISOLDE, the RFQCB will be tested in a new test bench constructed at ISOLDE off-line laboratory [7] for this purpose. Before and after ISCOOL, diagnostics will be placed for the characterization of the ion beam.

In fig. 2, the layout of the test bench is shown. The ion beam will be produced by an ion source (see next 


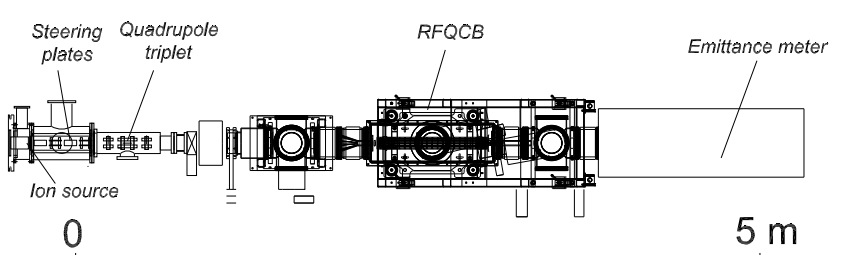

Fig. 2. Layout of the test bench for the RFQCB verification and optimization.

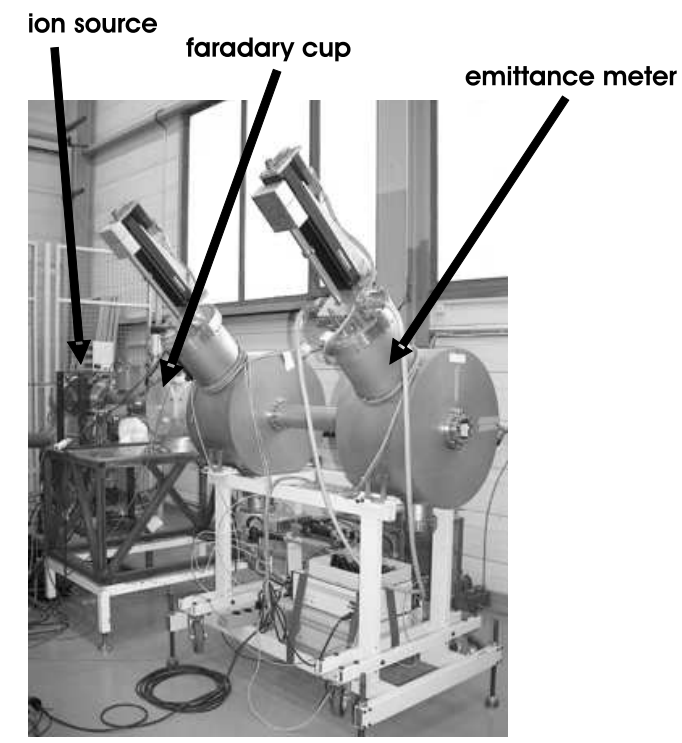

Fig. 3. Picture of the test bench for the ion source measurements.

paragraph), and directly transported to the RFQCB. Up and downstream the RFQCB diagnostic devices will be placed to characterize the main parameters of the machine. For example, transmission tests depending on buffer gas pressure, RF frequency or RF voltage amplitude.

The ion source installed in the test bench is a plasma ion source from the MISTRAL experiment [8]. It is able to provide ion beams either of alkali elements (mainly potassium) acting as a surface ionization source or gas elements as a plasma ion source. The maximum beam energy is $80 \mathrm{keV}$ with $60 \mathrm{keV}$ used for normal operation. Downstream the ion source, four pairs of steering plates (two for the longitudinal steering and two for the vertical) allow to displace the beam before it enters the quadrupole triplet placed just afterwards for focusing of the beam (injection into the RFQCB).

In parallel to the construction of the RFQCB, the ion source and an emittance meter have been coupled: firstly, to verify the correct operation of the source either in surface and gas mode; secondly, to produce figures for the emittance in both phase space planes $\left(x, x^{\prime}\right)$ and $\left(y, y^{\prime}\right)$ for the transmission tests of ISCOOL; finally, to find out the proper settings to assure that the shape of the phase space in the emittance meter is the same that would be in the location of the ISOLDE beam line where the RFQCB will be installed [2]. Figure 3 shows a picture of the line for the optics optimization of the beam from the ion source.

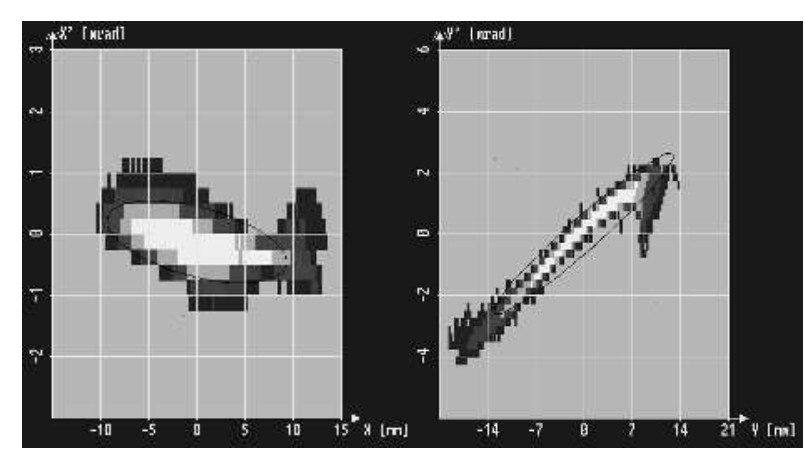

Fig. 4. Example of emittance plots from ion source of the test bench: horizontal phase spaces coordinates $\left(x-x^{\prime}\right)$ (left) and vertical $\left(y-y^{\prime}\right)$ (right).

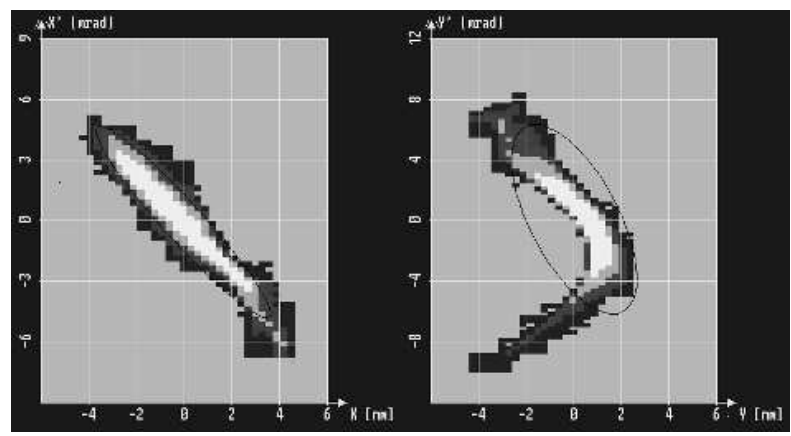

Fig. 5. As fig. 4 for a beam better focused (note difference scales).

Figure 4 illustrates original emittances from an ion source in $\left(x, x^{\prime}\right)$ and $\left(y, y^{\prime}\right)$ planes. After tuning the beam with the quadrupole triplet, emittances in both planes were better focused, as shown in fig. 5 . All emittance plots were measured for an alkali beam at $60 \mathrm{keV}$. The mean value obtained for the measurements of the transverse geometrical emittance enclosing $90 \%$ of the beam $\left(\epsilon_{60 \mathrm{keV}}^{90 \%}\right)$ is around $6.5 \pi \mathrm{mm} \cdot \operatorname{mrad}$ in $\left(x, x^{\prime}\right)$ and $\left(y, y^{\prime}\right)$.

We acknowledge the collaboration of LMU and University of Mainz in the construction of the RFQCB. One of us (A.J.) acknowledges the support from the Academy of Finland.

\section{References}

1. E. Kugler, Hyperfine Interact. 129, 23 (2000).

2. A. Jokinen et al., Nucl. Instrum. Methods Phys. Res. B 204, 86 (2003).

3. I. Podadera, CERN-AB-NOTE-2004-062 (2004).

4. A. Nieminen et al., Nucl. Instrum. Methods Phys. Res. A 469, 244 (2001).

5. F. Herfurth et al., Nucl. Instrum. Methods Phys. Res. A 469, 254 (2001).

6. I. Podadera et al., Nucl. Phys. A 746 C, 647 (2004).

7. www.cern.ch/isolde-offline.

8. M.D. Lunney et al., Hyperfine Interact. 99, 105 (1996). 